\title{
Pedestrian Detection in Poor Visibility Conditions: Would SWIR Help?
}

\author{
Massimo Bertozzi ${ }^{1}$, Rean Isabella Fedriga ${ }^{1}$, Alina Miron ${ }^{2}$, \\ and Jean-Luc Reverchon ${ }^{3}$ \\ 1 Dipartimento di Ingegneria dell'Informazione, Università di Parma, Italy \\ \{bertozzi,fedriga\}@vislab.it \\ www.vislab.it \\ 2 INSA de Rouen, 685 Avenue de l'Université, \\ 76801 Saint-Étienne-du-Rouvray Cedex, France \\ alina.miron@insa-rouen.fr \\ www. insa-rouen.fr \\ 3 III-V Laboratoire, Route de Nozay, F-91461 Marcoussis Cedex, France \\ jean-luc.reverchon@3-5lab.fr \\ www. 3-5lab.fr
}

\begin{abstract}
The 2WIDE_SENSE (WIDE spectral band \& WIDE dynamics multifunctional imaging SENSor Enabling safer car transportation) EU funded project is aimed at the development of a low-cost camera sensor for Advanced Driver Assistance Systems (ADAS) applications able to acquire the full visible to Short Wave InfraRed (SWIR) spectrum from 400 to $1700 \mathrm{~nm}$. This paper presents the first results obtained by investigating the SWIR contribution to pedestrian detection in difficult visibility conditions as haze and fog employing the wide-bandwidth camera developed within the project.
\end{abstract}

Keywords: SWIR, pedestrian detection, classification, large bandwidth cameras, haze, fog.

\section{Introduction}

Although several improvements in vehicle safety have been achieved in the last 25 years (e.g. crash tests, passive safety measures, new energy absorption materials, etc.), further reductions in road fatalities and injuries must be achieved.

The development of active video-based driver assistance systems to detect pre-emptively dangerous situations involving Vulnerable Road Users (VRU) as pedestrians is thus of fundamental importance for warning the driver or automatically taking control of the vehicle (e.g. braking) and becomes particularly valuable in case of driver's distraction or poor visibility conditions. Yet visionbased pedestrian detection is a difficult problem for a number of reasons 2. The objects of interest appear in highly cluttered backgrounds and have a wide range of appearances, due to body size and pose, clothing and outdoor lighting conditions. Because of the moving vehicle, one does not have the possibility to 

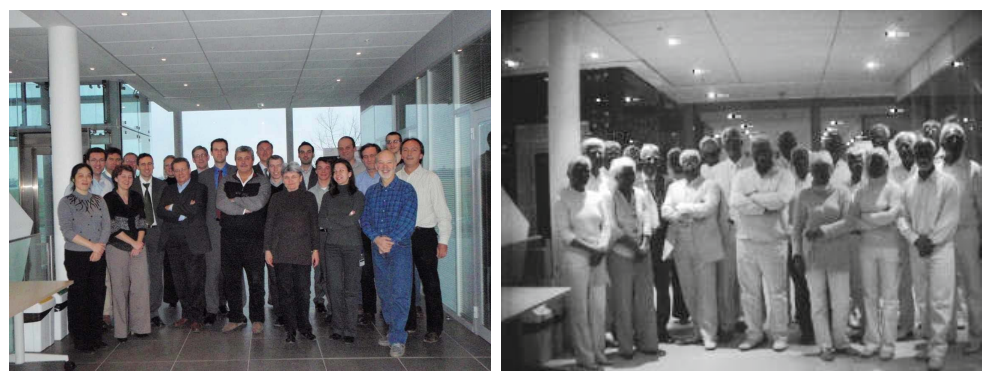

Fig. 1. Indoor images: in the SWIR spectrum people's hair and skin appear very different from how we are used to see them in everyday life

use simple background subtraction methods (such as those used in surveillance applications) to obtain a foreground region containing the human shape. Furthermore, pedestrians can exhibit highly irregular motion, making prediction and situation analysis difficult. Finally, there are hard real-time requirements and tight performance criteria.

Imaging in the Short Wave InfraRed (SWIR) can bring useful contrast to situations and applications where visible or thermal imaging cameras are ineffective making SWIR frequently used for diverse applications such as aligning telecommunications fibers and sources, engineering optical wave-guides, inspecting pharmaceutical quality, sorting recycled plastics, monitoring incoming sources of raw agricultural products to groom out contamination by dirt, stones or packaging debris, as well as grade sorting by moisture level or fat content [8]. Unlike Mid Wave IR (MWIR) and Long Wave IR (LWIR), SWIR cameras can image through the windshield and thus be mounted in the vehicle's cabin for a "driver's eye" view of the way ahead. Moreover SWIR imagers have the ability to see farther through haze than visible imagers due to the reduced scattering as the wavelength of light gets longer. Long distance imaging through the atmosphere is clearer too, making SWIR suitable for investigations in the field of automotive applications [12 the main issues concerning it having been to achieve low cost SWIR sensors, operating at close to room temperature and CMOS compatible. In the last few years though the standard technology based on indium bump hybridization has progressively decreased its pixel pitch going to VGA or XVGA format mainly available in the US. Nevertheless, most of VGA format were based on the $25 \mu \mathrm{m}$ pitch from FLIR Indigo ISC0002 readout circuit which consists in an expensive device not compatible with the automotive market. Work done within the EU funded 2WIDE_SENSE project has involved a $15 \mu \mathrm{m}$ pitch -the best format to replace the VGA format- reaching a low cost hybridization process and packaging, and thus making the SWIR imager obtained most suitable for the automotive market requirements. Purpose of this article is to illustrate our tests done within the 2WIDE_SENSE project to explore the characteristics of SWIR images for the VRU detection application in difficult visibility conditions, specifically haze and fog. 
A peculiar characteristic to be noted about the SWIR spectrum is that human skin, having a very high water content, absorbs much of the longer wavelengths appearing in SWIR images very dark if not almost black (see fig. 1). Previous works within the IR bandwidth have dealt with skin detection both for face recognition [4] or people detection [3,9,11] but they are ineffective for an automotive pedestrian detector being very little the skin area usually showing from the clothes. We have therefore applied a classic approach for pedestrian detection, an SVM classifier based on deformable part models [6,7].

This paper is organized as follows: section 2 describes the $\mathrm{HW}$ equipment employed, section 3 describes the experimental measurements carried out to test the SWIR wavelength bandwidths characteristics for VRU's detection, section 4 reports the results obtained, and section 5 states our final considerations.

\section{Hardware Equipment}

The European funded 2WIDE_SENSE collaborative project has the aim of developing a low-cost camera able to acquire in the full visible to SWIR bandwidth. This task required the development of a specific sensor and of a large bandwidth lens. In addition, the camera is featuring a filter on the sensors that enables the independent acquisition of 4 different spectral bandwidths.

The sensor of the 2WIDE_SENSE camera module has been mainly developed by Alcatel-Thales III-V Lab and is an uncooled In GaAs and InP-based $640 \times 512$ pixels array with a $15 \mu \mathrm{m}$ pitch and a MAGIC ${ }^{\mathrm{TM}}$ logarithmic readout circuit (see fig. 2). The two main features of the sensor are the large spectrum sensitivity (400-1700 $\mathrm{nm}$ ) and the logarithmic gain that enables to avoid saturation effects.

Furthermore a specific micro-lens module has been developed by the OPTEC s.p.a (see fig. 2) to let the camera exploit the full spectrum capabilities. The OB-V-SWIR_16 apochromatic lens is based on a combination of six elements produced using a specific moldable glass. The lens transmittance is nearly the same in the whole functioning band: $>98 \%$ in the $400-1550 \mathrm{~nm}$ interval and decreasing down to $96 \%$ in the $1550-1700 \mathrm{~nm}$.
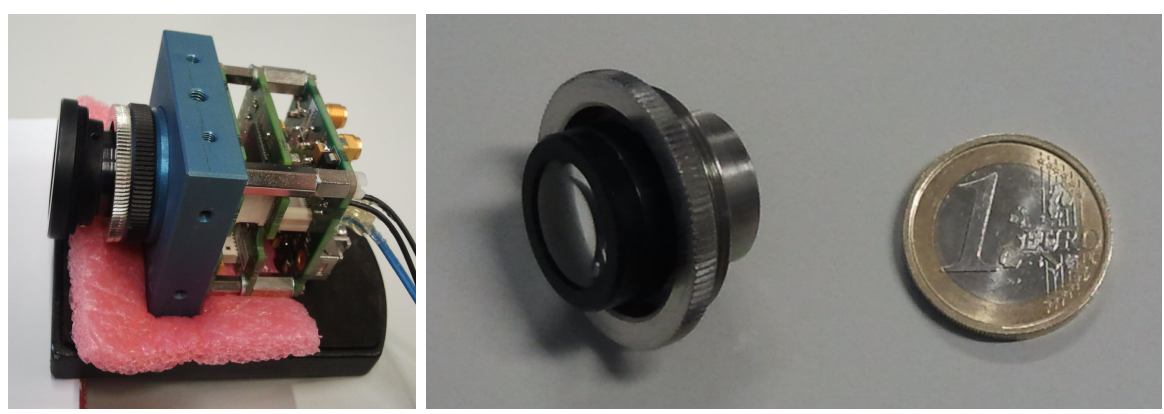

Fig. 2. The 2WIDE_SENSE camera module and the OB-V-SWIR_16 micro-lens 


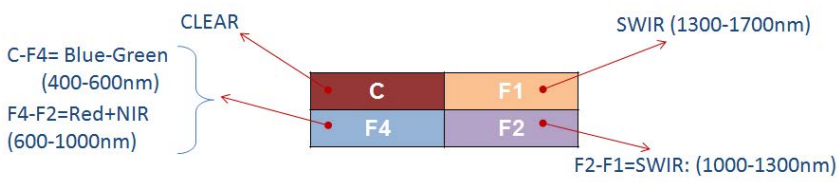

Fig. 3. The $4 \times 4$ filter mask applied on the FPA

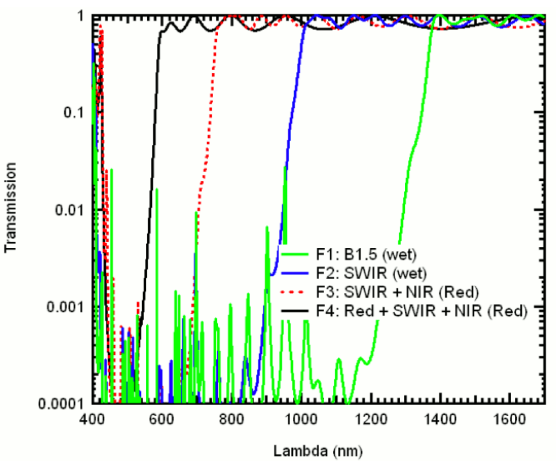

Fig. 4. The four filters transmission bands (filter F3 has eventually been discarded)

The most interesting feature of the developed camera is the presence of a Bayer-like filter to independently acquire specific spectral bandwidths that have been selected according to automotive world needs. More precisely, four different sapphire substrates have been grown on the pixel array and fourteen layers of $\mathrm{TiO}_{2}$ and $\mathrm{SiO}_{2}$ have been deposited on the substrates obtaining a $4 \times 4$ pixels mask pattern (see fig. 3). Each pixel-filter is a high-pass filter with the following bandwidths: $C$ clear (no filter, full bandwidth), $F 1$ 1350-1700 $\mathrm{nm}$ (SWIR), F2 1000-1700 $\mathrm{nm}$ (SWIR), and F4 >540 $\mathrm{nm}$ (Red, NIR, and SWIR) (see fig. 4).

The bandwidths of each filter have been selected according to most used ADAS functions like Traffic Sign Recognition and High Beam Assist. Anyway other bandwidths can be easily obtained combining different contributions; as an example the blue and green bandwidths can be obtained as a difference between the $C$ component and the $F 4$ contribution.

The large bandwidth camera module developed during the project has been available and therefore only tested during the final stage of the 2WIDE_SENSE experiments. This article reports about the preliminary tests done using a stateof-the-art In GaAs camera module with the OB-V-SWIR_16 micro-lens and highpass SWIR filters applied on the lens (transmission bands as F1 and F2).

\section{$3 \quad$ Field Tests}

A database of more than 10,000 images in different illumination and weather conditions with varied combinations of gain and exposure time has been 


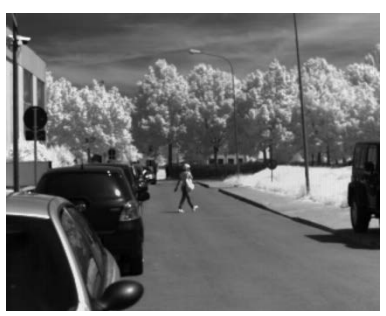

Clear

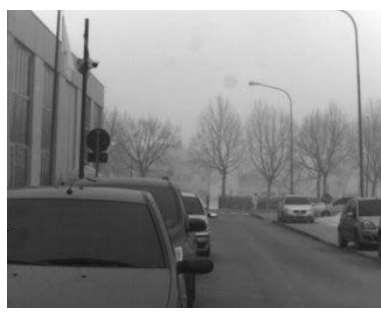

Haze

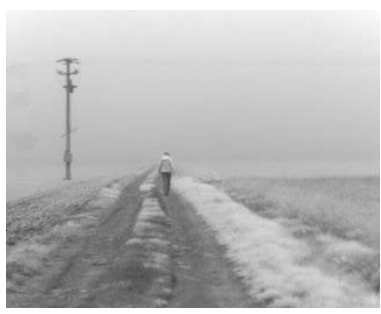

Fog

Fig. 5. Images acquired in the SWIR spectrum $(1.0$ - $1.7 \mu m)$-filter F2-with different visibility conditions during summer, autumn and winter respectively

collected, paying special attention to cases of reduced visibility caused by haze and fog (see fig. [5).

In order to get a complete picture of the SWIR contribution to visibility, we have carried out acquisitions both in different bandwidths during similar illumination and weather conditions and in the same bandwidth with different illumination and weather conditions. Some examples are discussed in fig. 6 .

\section{Results}

A thorough investigation of images acquired in the SWIR bandwidths and comparisons with images acquired in the visible spectrum have been carried out. In the following subsections three visibility conditions will be dealt with: clear sky, haze and fog. To detect pedestrians in the SWIR spectrum the object detection method based on deformable part models illustrated in [7] and [6] has been employed. Being based on both contrast sensitive and contrast insensitive HOG features, we have found that training the classifier on visible images only was suitable for detections on SWIR images with comparable detection rates. The following results have therefore been obtained training the classifier on datasets publicly available on the web (the PASCAL datasets [5]) featuring images acquired in the visible spectrum only.

\subsection{Clear}

Images acquired in the SWIR spectrum with clear sky conditions show that high water content objects like the human skin appear a lot darker than in visible only images. Nonetheless this peculiar characteristic is of difficult utility to detect pedestrians as skin areas showing from the clothes of a person are of variable sizes and positions. In addition, image contrast may change significantly in different seasons with light changes or particular atmospheric conditions such as high humidity levels and other sorts of absorption phenomena, making the skin color an unreliable indicator for automotive applications (see fig. 7). 

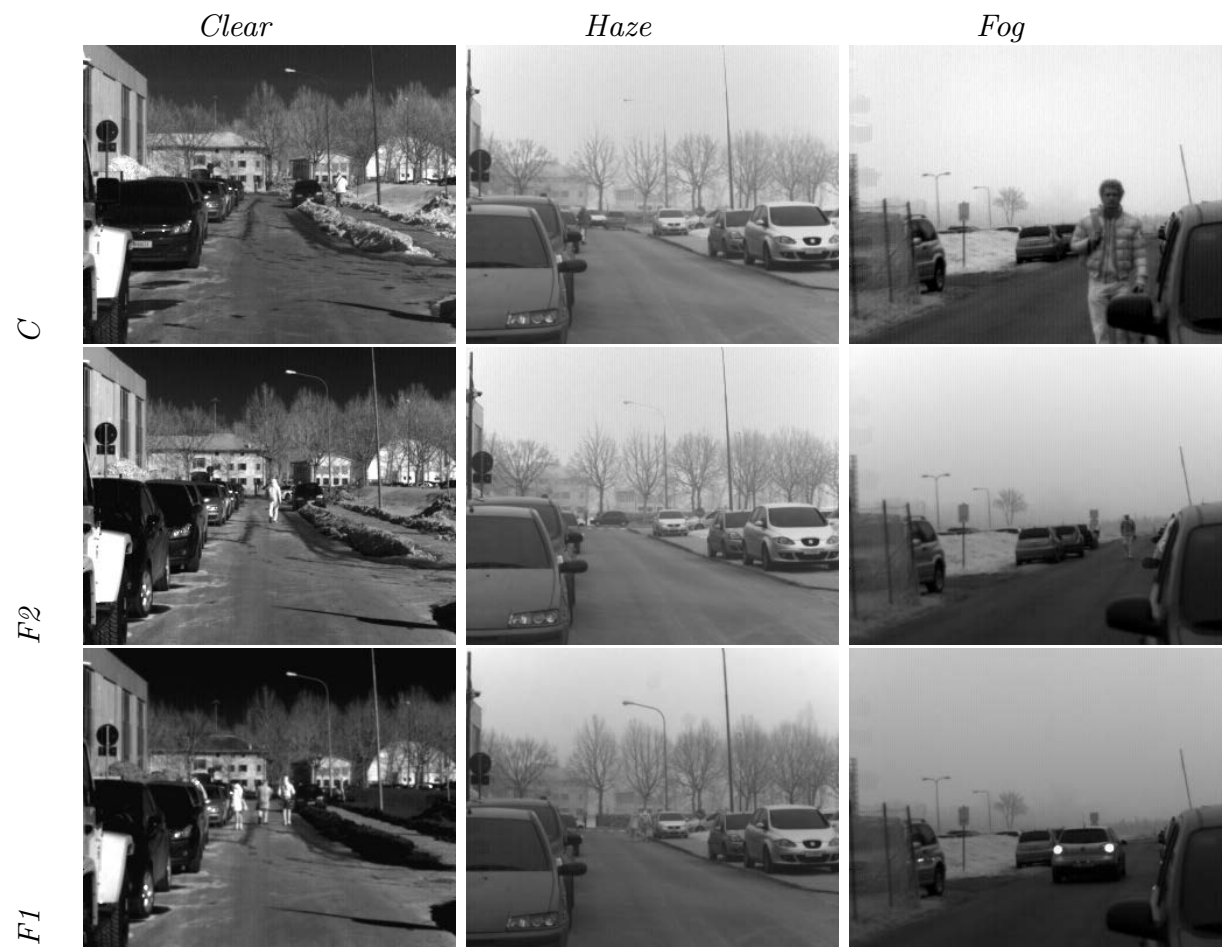

Fig. 6. Images acquired in the whole visible to SWIR spectrum $(0.4-1.7 \mu m)$-filter $C$ - and in the two SWIR bandwidths available on the FPA: SWIR spectrum (1.0 $1.7 \mu \mathrm{m})$-filter F2- and SWIR spectrum $(1.3-1.7 \mu \mathrm{m})$-filter F1-. Acquisitions have been carried out with clear, hazy and foggy visibility conditions, as indicated by the columns titles, and during different seasons, this having as a consequence different gain and exposure time settings so to avoid image saturation. In particular, images shown in the first column appear quite dark in respect of the others as due to a very bright winter day the exposure time had been set to $1.0 \mathrm{msec}$. Note that, as stated by the theory of major water absorption happening in the SWIR spectrum, in these images the snow color (low right side, adhering to the footpath curb) gets darker as the bandwidth narrows from the whole visible to SWIR spectrum to the pure SWIR ones.
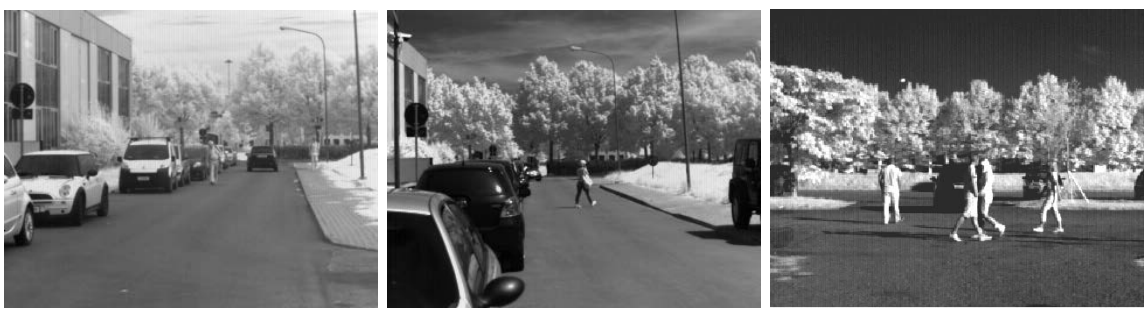

Fig. 7. Images acquired with SWIR filter F2 in different light conditions 

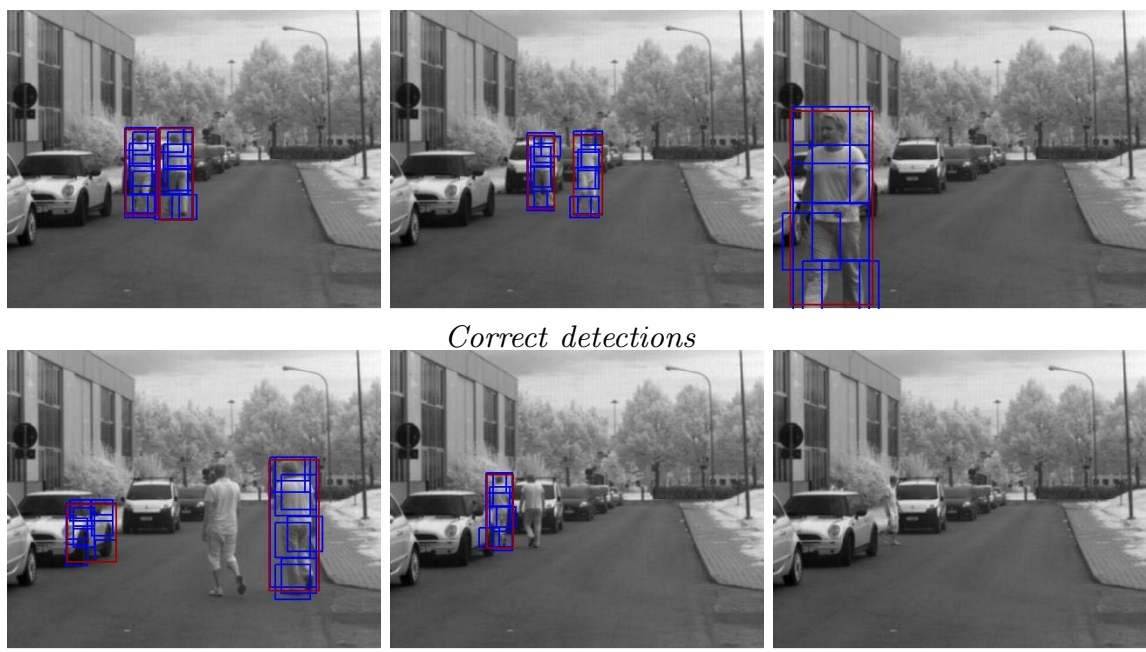

Correct detections
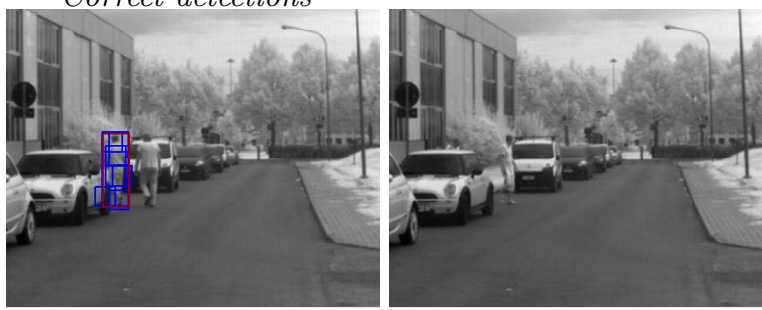

Miss and incorrect detections

Fig. 8. Detection examples employing the classifier on images acquired with filter F2

Through the classification process, correct detection values comparable to those obtained on visible only images are achievable but with no practical advantage by employing a SWIR sensor in respect of a standard visible only one (see fig. 8). Results of the classification and comparisons between different classifiers on SWIR images will be presented [1].

\subsection{Haze}

Haze is an atmospheric phenomenon where dust, smoke and other wet or dry particles obscure the sky's clarity. The SWIR wavelengths are able to penetrate those particles layer, making visibility clearer at distance (see fig. 9). However, due to the space between particles, haze becomes perceptible only from kilometers afar making a pedestrian detection application of questionable utility.

Fig. 10] images report a visibility condition where the water particles layer is denser and closer to the observer than haze but not as thick and close as fog usually is. The house in the background, so as the pedestrians half way from it, are all quite visible but the pedestrians already too small, so that their eventual detection would be of minor use for an automotive application.

\subsection{Fog}

Acquisitions carried out in fog conditions have shown that, despite the longer wavelengths capability of penetrating water particles, a clear visibility through the fog is not achievable employing a SWIR sensor (see fig. 11). Due to the 


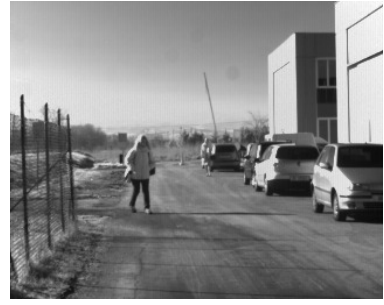

visible to SWIR

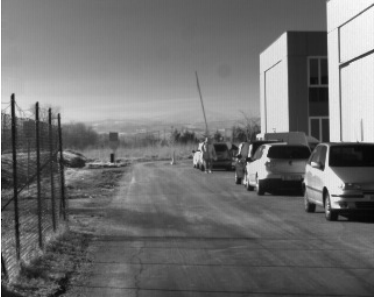

SWIR

Fig. 9. Images acquired in the whole visible to SWIR $(0.4-1.7 \mu \mathrm{m})$ spectrum - filter $C$ and in the SWIR only $(1.3-1.7 \mu \mathrm{m})$ spectrum -filter $F 1$ - respectively: almost invisible in the first image, the backgorund mountains become quite clear in the second image
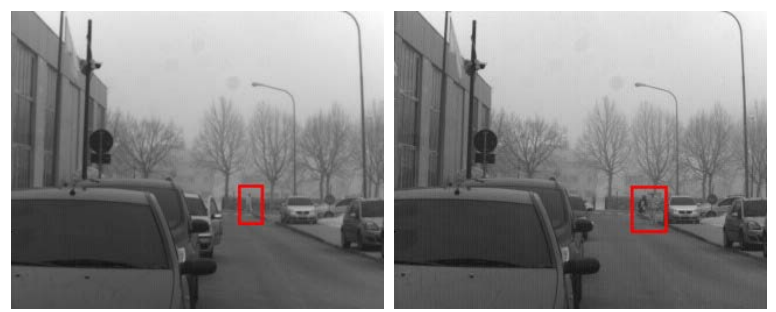

Fig. 10. Far away pedestrians, manually surrounded by a red bounding box in these images acquired with filter $F 1$

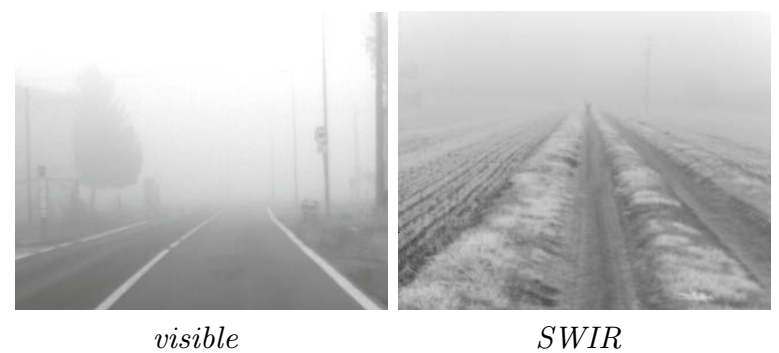

Fig. 11. Images acquired in the visible only $(0.4-0.9 \mu \mathrm{m})$ and SWIR $(1.0-1.7 \mu \mathrm{m})$ spectrum -filter $F 1$ - respectively: with similar fog densities the scattering effect is predominant at both bandwidths so that the pedestrian walking along the country path in the SWIR image is hardly detectable

peculiar nature of fog the scattering effect, predominantly in the forward direction, affects the SWIR wavelengths making imaging at distance impossible. The classifier gives correct detections only when the pedestrian is close enough to the camera (see fig. 12). 

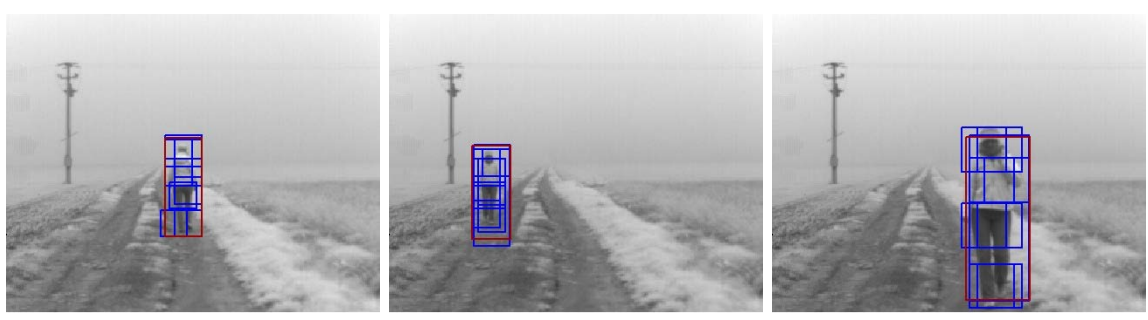

Correct detections

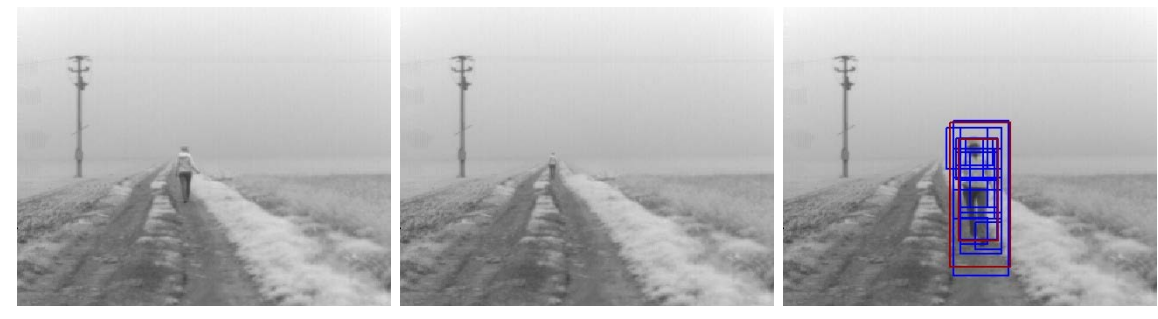

Miss and incorrect detections

Fig. 12. Detection examples employing the classifier on images acquired with filter F2

\section{Conclusions}

Light scattering effects occur daily in the atmosphere: while some wavelengths are absorbed by air molecules, others are scattered. When water vapor and dust are present in the atmosphere, additional effects are observed so that most of the visible wavelengths are being scattered to the ground observers eyes. As the particles get denser and their size increases, visible imaging of any distance becomes very difficult. Longer wavelengths, as the SWIR ones, penetrate the haze providing clearer details of what appears hidden in a visible image. But the phenomenon of scattering is complex, made up of reflection, refraction and diffraction with variations such as forward, side, back scatter, and it is dominated by diffraction as the light diffracts or bends around molecules or particles. Water vapor and carbon dioxide have many infrared absorbance bands but in the short wave there is a mixture of absorbance bands and transmission windows permitting SWIR imagers to see for long distances with little loss due to either scattering or absorbance. As the particle size grows to a diameter or cross section equal or larger than the wavelength, the scattering is predominately more forward in direction and the intensity loss also becomes less dependent on wavelength. Eventually the size of fog, dust or smoke particles become large enough to obscure SWIR images and even the longer wavelengths are easily scattered [10].

When light interacts with atoms and molecules that are much smaller than the wavelength of light, light rays are scattered (at a rate of $(1 / \text { wavelength })^{4}$ ) in the phenomenon of Rayleigh scattering, thus preventing short wavelengths from travelling long distances. As light encounters spherical particles of dimensions near to or larger than that of its wavelength, it is attenuated at the slower rate of 1 /wavelength, as predicted by the Mie theory of scattering [8]. 
Our investigations on images acquired in different spectra going from the only visible to the only SWIR one, have demonstrated that reduced visibility phenomena as haze and fog feature quite different behaviors in the SWIR spectrum but still of no practical utility for automotive applications. Whereas better visibility through haze may be achieved by employing SWIR sensors but is of negligible benefit for pedestrian detection being haze a long distance phenomenon, no improvements can be obtained in foggy conditions, leaving thermal imagers (Far InfraRed wavelengths) as the most effective technology currently available for this purpose.

Acknowledgments. The work described in this paper has been developed in the framework of the 2WIDE_SENSE (WIDE spectral band \& WIDE dynamics multifunctional imaging SENSor Enabling safer car transportation) Project funded by EU within the FP7 Seventh Framework Programme.

\section{References}

1. Bertozzi, M., Fedriga, R.I., Miron, A., Reverchon, J.-L.: SWIR vs. Visible Imagers for Pedestrian Detection in Reduced Visibility Conditions. In: Procs. IEEE Intl. Conf. on Intelligent Transportation Systems, The Hague, Nederlands (submitted)

2. Binelli, E., Broggi, A., Fascioli, A., Ghidoni, S., Grisleri, P., Graf, T., Meinecke, M.-M.: A Modular Tracking System for Far Infrared Pedestrian Recognition. In: Procs. IEEE Intelligent Vehicles Symposium 2005, Las Vegas, USA, pp. 758-763 (June 2005)

3. Brooks, A.L.: Improved Multispectral Skin Detection and Its Application to Search Space Reduction for Dismount Detection Based on Histograms of Oriented Gradients. Master's thesis, Air Force Institute of Technology, Wright-Patterson Air Force Base, Ohio, USA (October 2012)

4. Chang, H., Koschan, A., Abidi, M.: Multispectral visible and infrared imaging for face. In: Procs. IEEE Computer Vision and Pattern Recognition Workshops, pp. 1-6. IEEE Computer Society, Anchorage (2008)

5. Everingham, M., van Gool, L., Williams, C., Winn, J., Zisserman, A.: The Pascal visual object classes, http://pascallin.ecs.soton.ac.uk/challenges/VOC

6. Felzenszwalb, P.F., Girshick, R.B., McAllester, D.: Cascade object detection with deformable part models. In: Procs. IEEE Conf. on Computer Vision and Pattern Recognition, pp. 2241-2248 (2010)

7. Felzenszwalb, P.F., Girshick, R.B., McAllester, D., Ramanan, D.: Object detection with discriminatively trained part-based models. IEEE Trans. on Pattern Analysis and Machine Intelligence 32(9), 1627-1645 (2010)

8. Hansen, M.P., Malchow, D.S.: Overview of SWIR detectors, cameras, and applications. In: Procs. SPIE, vol. 6939, Thermosense XXX (March 2008)

9. Kilgore, G.A., Whillock, P.R.: Skin Detection Sensor, United States Patent Office, Publication nr. US2007/0106160A1, Application n. 11/264,654, Issued patent US7446316, 2008-11-04 (November 2008)

10. Malchow, D.: NIR Trends: Penetrating The Haze Of Scattered Light. In: UTC Aerospace Systems (Sensors Unlimited Products) Goodrich Corporation (October 2008)

11. Nunez, A.S., Mendenhall, M.J.: Detection of Human Skin in Near Infrared Hyperspectral Imagery. In: Procs. IEEE Geoscience and Remote Sensing Symposium, pp. 621-624. IEEE Computer Society (July 2008)

12. Valldorf, J., Gessner, W. (eds.): Advanced Microsystems for Automotive Applications 2005. Springer, Berlin (2006) 